\title{
Detection of nucleated red blood cells using the Mindray BC-6800Plus hematology analyzer: a clinical performance evaluation
}

\author{
Nengyong Wang, Junyao Jiang, Ji'an Wang, Yao He, Ning Wen, Yulin Liu, Yashu Wang, Haijun Li, \\ Ping Shi \\ Laboratory Department, Guangyuan Central Hospital, Guangyuan, China \\ Contributions: (I) Conception and design: N Wang, J Jiang; (II) Administrative support: P Shi; (III) Provision of study materials or patients: J Wang, \\ Y He, N Wen; (IV) Collection and assembly of data: Y Liu, Y Wang, H Li; (V) Data analysis and interpretation: N Wang, J Jiang; (VI) Manuscript \\ writing: All authors; (VII) Final approval of manuscript: All authors. \\ Correspondence to: Ping Shi. Laboratory Department, Guangyuan Central Hospital, Guangyuan 628017, China. Email: 3258045559@qq.com.
}

\begin{abstract}
Background With the development of instrument technology, the functions and detection methods of automatic blood cell analyzers have become more complex. To ensure optimal clinical applicability, it is crucial to select an automatic blood cell analyzer with excellent clinical detection performance. This study evaluated the latest Mindray BC-6800Plus automatic blood cell analyzer and assessed its performance in the detection of nucleated red blood cells (NRBCs).

Methods: A total of 490 clinical blood samples were used to assess the performance of the instrument, including parameters such as precision, linearity, conformity rate of manual microscopic examination, carryover, and limit of quantitation.

Results: The instrument showed a small carryover $(\leq 0.02)$ and excellent linearity $\left(\mathrm{R}^{2} \geq 0.9986\right)$. The reproducibility of the sample tests was satisfactory, and the coefficient of variation (CV) of the test results [0.98-1.72\% and 0.62-6.97\% for white blood cells (WBCs) and NRBCs, respectively] were significantly lower than that declared by the manufacturer (2.5\% and $20 \%$ for WBCs and NRBCs, respectively). Thus, the BC-6800Plus satisfies the requirements of clinical testing. Two separate Mindray BC-6800Plus machines were tested and found to be in good agreement with each other and with manual microscopy methods. Furthermore, WBC and NRBC counts were highly consistent with results obtained using the XN-9100 blood analyzer.
\end{abstract}

Conclusions: The Mindray BC-6800Plus is an excellent analyzer that can provide timely and accurate reports for clinical laboratory detection of NRBC.

Keywords: Hematology analyzer; nucleated red blood; parameter; manual microscopic examination

Submitted May 25, 2021. Accepted for publication Aug 11, 2021.

doi: 10.21037/apm-21-1772

View this article at: https://dx.doi.org/10.21037/apm-21-1772

\section{Introduction}

Combined with a patient's clinical symptoms and history, routine peripheral blood examinations play a vital role in the clinical diagnosis and treatment of many diseases, especially diseases involving the blood system (1). For example, hemoglobin (HGB) levels and platelet (PLT) counts can determine whether a patient needs blood transfusions, the number of white blood cells (WBCs) can indicate the degree of infection, and the appearance of abnormal cells may be closely related to diseases of the circulatory system and tumors (2-4). Furthermore, the presence of nucleated red blood cells (NRBCs) is closely associated with many serious diseases. Therefore, methods that can accurately and efficiently analyze peripheral blood are of great clinical 


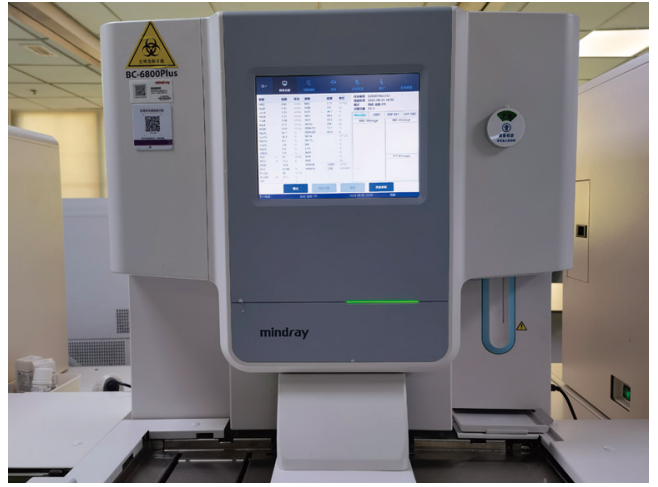

Figure 1 The latest Mindray BC-6800Plus automatic blood cell analyzer.

significance in the diagnosis and treatment of diseases (5).

While manual microscopy is an established reference method for blood cell counts, it has a huge requirement for experienced manpower, and has low precision and efficacy, and is thus not suitable for the clinical setting $(6,7)$. Automatic blood cell analyzers can combine resistance with flow cytometry to ensure accuracy and repeatability of clinical specimen results, and thus may be more suitable for clinical applications. It can also screen clinical specimens with abnormal classifications, abnormal numbers, and abnormal cells $(1,8)$. With advancements in instrument technology, the function of new blood cell analyzers has significantly increased, and the structure and principles are also more complex. To ensure that the performance of an automatic blood cell analyzer meets the clinical requirements, many committees and research institutes have developed standards and guidelines to facilitate the evaluation of instrument parameters and performance $(5,7)$.

Mindray BC-6800Plus (Mindray, Shenzhen, China) is the latest generation of automatic hematology analyzer (Figure 1), which can rapidly assess both outpatient and emergency patient samples. This study evaluated the performance of the Mindray BC-6800Plus by examining its precision, linearity, conformity rate of manual microscopic examination, carryover, and limit of quantitation $(9,10)$. In addition, the performance was compared with other brands of blood cell analyzers, namely, the Sysmex XN-9100. In addition to neonates 1 week after birth, the presence of nuclear erythrocytes in peripheral blood is mostly related to the pathological state, so the accurate detection of nuclear erythrocytes is very important. The Mindray BC-6800Plus showed superior performance in detecting the presence of NRBCs in neonates compared to other blood cell analyzer systems.
We present the following article in accordance with the MDAR reporting checklist (available at https://dx.doi. org/10.21037/apm-21-1772).

\section{Methods}

\section{Samples and analyzer}

All procedures performed in this study involving human participants were in accordance with the Declaration of Helsinki (as revised in 2013). The study was approved by the Ethics Committee of the Guangyuan Central Hospital, Sichuan, China and informed consent was taken from all the patients. Blood samples were collected from outpatients and inpatients at the Guangyuan Central Hospital between December 2019 and May 2020, and stored in BD Vacutainers (reference 367861, Franklin Lake, NJ, USA) with potassium ethylenediamine tetraacetate $(1.5-2.2 \mathrm{mg} / \mathrm{mL})$ to maintain anticoagulation. Samples showing obvious hemolysis, clotting, or developed clots were excluded. A total of 490 venous specimens were collected in this study. The presence of NRBCs in the blood samples was evaluated using two Mindray BC6800Plus auto hematology analyzers, using the $\mathrm{CD}$ [complete blood cell $(\mathrm{CBC})+$ differential (DIFF)] mode. All blood samples were evaluated within 8 hours of collection, during which the blood samples were stored at $18-26^{\circ} \mathrm{C}$. Evaluated CBC parameters included absolute WBC count $\left(\times 10^{9}\right.$ cells/L), absolute red blood cell (RBC) count $\left(\times 10^{12}\right.$ cells/L), HGB $(\mathrm{g} / \mathrm{L})$, hematocrit $(\%)$, PLT count $\left(\times 10^{9} / \mathrm{L}\right)$, mean corpuscular volume (fL), mean corpuscular HGB (pg), mean corpuscular HGB concentration $(\mathrm{g} / \mathrm{L})$, and $\mathrm{RBC}$ distribution width (\%). Percentage and absolute counts for neutrophils, lymphocytes, monocytes, eosinophils, and basophils were also assessed. The morphology of the CBC was examined using morphological markers of WBCs, RBCs, and PLT.

\section{Precision}

Repeatability refers to measurements made using blood samples from many different healthy subjects, covering a range of measurements (AMR) $(11,12)$. Repeatability was determined by assessing each clinical patient sample more than 10 times and calculating the standard deviation (SD) and coefficient of variation (CV). The betweenrun precision was measured by quality control products with the same batch number provided by the instrument manufacturer. The same operator carried out quality control tests on two BC-6800Plus units every day for more than 
20 working days. The reproducibility was assessed using three levels of BC-6D controls according to the following design: 20 days $\times 2$ runs $\times 2$ replicates.

\section{Linearity}

Linearity is the ability to obtain test results within a given range that are proportional to the concentration of the tested substance in the sample (13). To verify this parameter, specimens with abnormal nuclear red cells were selected for analysis, and the parameters studied were WBC count and NRBC count. Whole blood samples with the highest and lowest values of NRBCs were selected for gradient dilution.

\section{A comparison of different methods}

The ability of the CD model of the Mindray BC-6800Plus to accurately identify NRBCs was compared with that of the CBC model of the XN-9100 machine (Sysmex).

\section{Conformity rate of manual microscopic examination}

Peripheral blood smears were prepared with Wright Giemsa reagent using the Mindray sc-120 automatic blood slide preparation device (14). The smears labeled with NRBC by the analyzer were manually counted by experienced laboratory technicians. Manual NRBC counts also included morphological analysis of WBCs, RBCs, and PLTs. The BC-6800Plus identifies and counts NRBCs in a dedicated DIFF/WNB channel by combining DIFF cell lysis, forward light scattering, lateral light scattering, and fluorescence emissions. The results are displayed as the percentage of WBC (NRBC per $100 \mathrm{WBC}$ ) and the absolute cell count. The correlation between the Mindray automatic counting results and the manual counting results was analyzed.

\section{Instrument comparability}

Two auto hematology analyzer BC-6800Plus machines were used to analyze the same samples, and instrument comparability was evaluated by examining the differences in the NRBC detection results obtained with the two analyzers.

\section{Carryover}

Carryover was evaluated using methods recommended by International Council for Standardization in Hematology (ICSH). The percentage of carryover for NRBCs and
WBCs was calculated using the following method (15):

$$
\text { Carryover } \%=L_{1}-L_{3} / H_{3}-L_{3}
$$

\section{Limit of quantitation}

To measure the detection limit of nucleated erythrocytes by the instrument, a sample with a concentration of five nucleated erythrocytes per $100 \mathrm{WBCs}$ was used for a gradient dilution until the concentration was $0.25 \%$, and each specimen was tested three times (16). The limit of quantitation of the instrument was the concentration of the specimen with a nucleated erythrocyte detection result of 0 .

\section{Statistical analysis}

Statistical analyses were performed using WPS 2021 and Graphpad 8.0 software. Paired Student's $t$-test was used to compare results obtained using BC-6800Plus and results obtained using manual microscopy, and between different blood samples. Passing-Bablok regression and BlandAltman analyses were used for method comparison between the BC-6800Plus and the Sysmex XN-9100 systems.

\section{Results}

\section{Precision}

A total of 10 venous whole-blood samples were analyzed on the BC-6800Plus machine and each sample was analyzed 10 consecutive times (Table 1). According to the manufacturers, the percentage $\mathrm{CV}$ in the detection of WBCs and NRBCs should be less than $2.5 \%$. If the WBC count exceeds $4 \times 10^{9} / \mathrm{L}$, the CV should be less than or equal to $20 \%$. The intra- and inter-operation precision were assessed using the Analysis-it software (Table 2).

\section{Carryover}

The BC-6800Plus parameters evaluated showed no significant carry forward. The CD mode carryover results of WBCs and NRBCs were $0.20 \%$ and $0.01 \%$, respectively.

\section{Linearity}

The parameters including WBCs, total NRBC numbers (NRBC\#), and percentage of NRBC (NRBC\%) showed good linear relationships at both high and median concentrations (Figure 2). WBC-D and WBC-N showed 
Table 1 The precision/reproducibility data for clinical samples on the BC-6800Plus analyzer

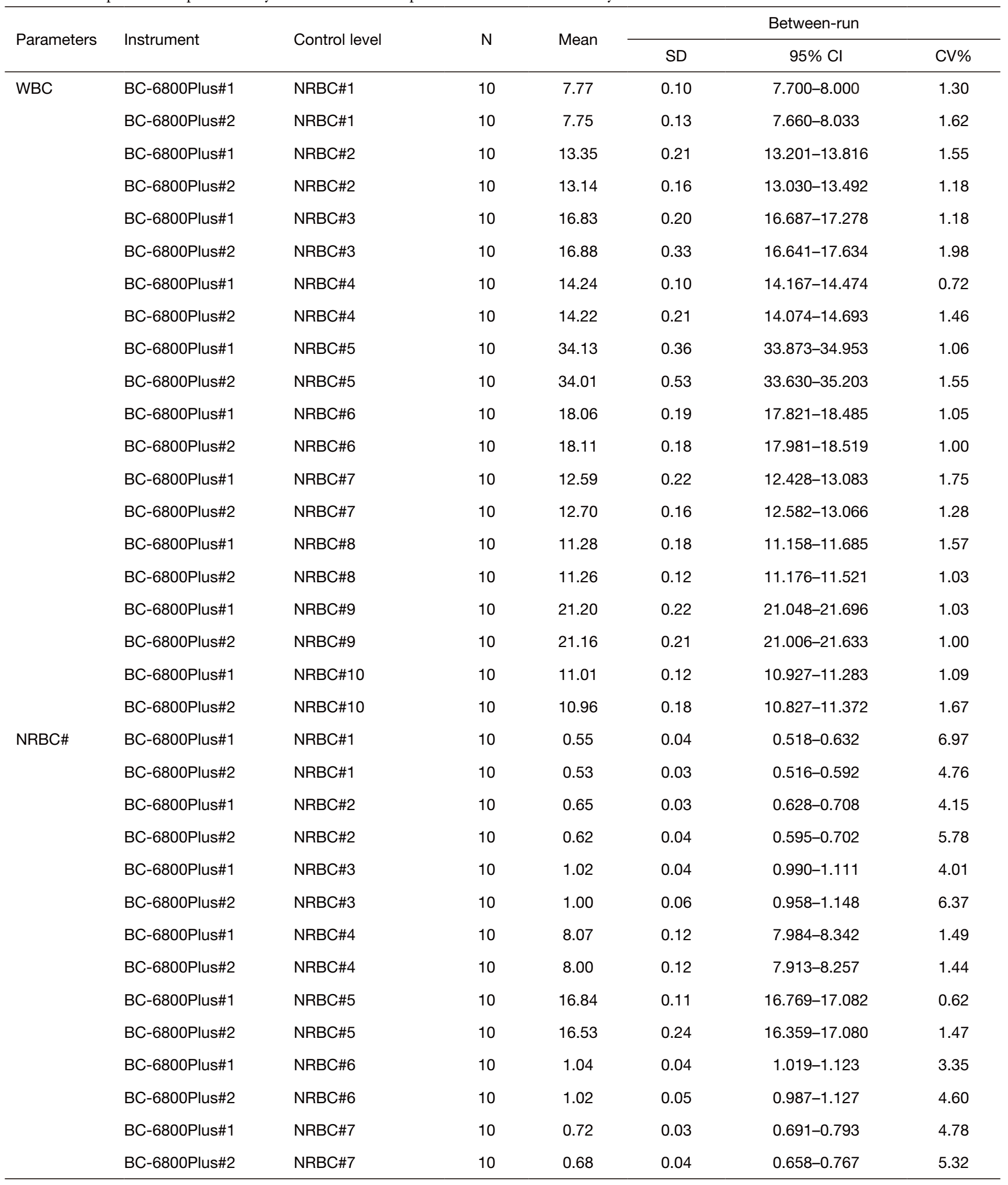

Table 1 (continued) 
Table 1 (continued)

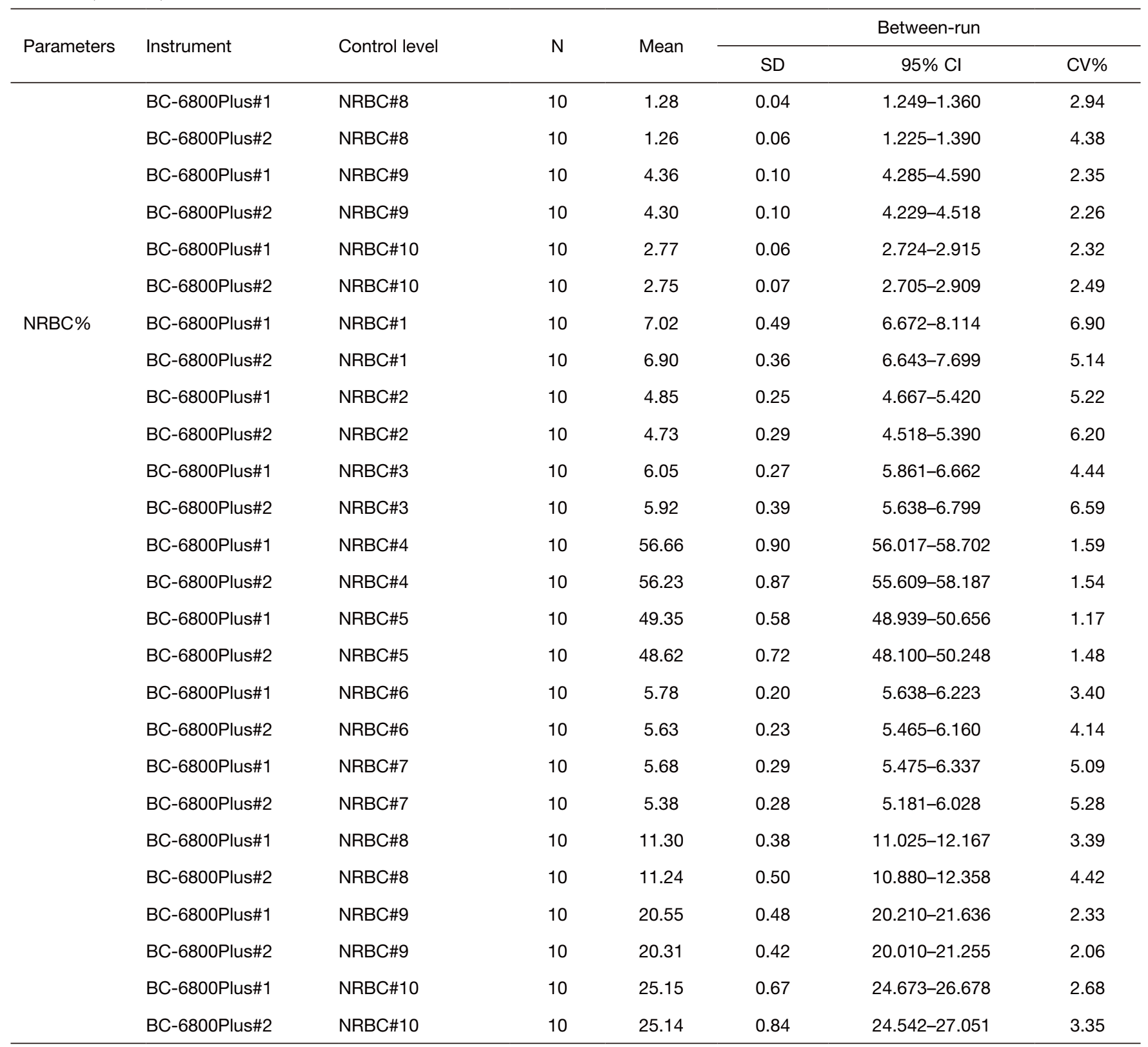

$\mathrm{SD}$, standard deviation; $\mathrm{Cl}$, confidence interval; CV, coefficient of variation; WBC, white blood cell; NRBC\#, number of nucleated red blood cells; NRBC\%, percentage of nucleated red blood cells.

excellent linearity in the range of $0.18-487.14 \times 10^{9} / \mathrm{L}$ $(\mathrm{r}=0.9996)$. The correlation coefficient of NRBC\# and NRBC\% was 1.005 and 1.003 , respectively.

\section{A comparison of different analyzer machines}

A total of 30 samples containing NRBCs were selected and analyzed using the Mindray BC-6800Plus and Sysmex XN9100 systems. The parameters for comparison included WBCs, NRBC\#, and NRBC\%. There was a high or very high correlation between the two devices in the WBC and NRBC parameters. The differences in each parameter were considered clinically insignificant in the Bland-Altman plot (Figure 3). 
Table 2 The precision/reproducibility data for BC-6D controls assessed s using the BC-6800Plus analyzer

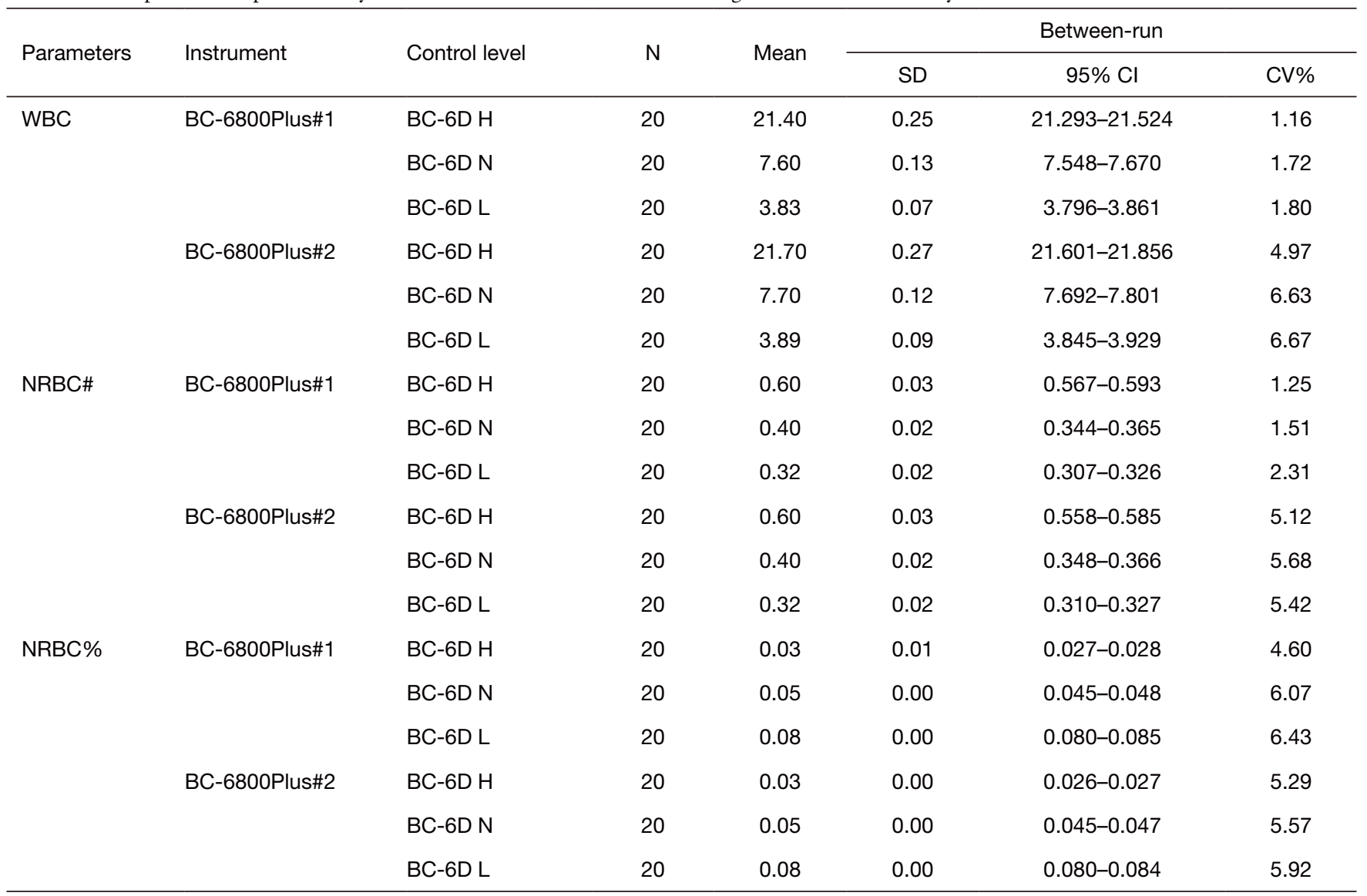

$\mathrm{SD}$, standard deviation; $\mathrm{Cl}$, confidence interval; CV, coefficient of variation; WBC, white blood cell; NRBC\#, number of nucleated red blood cells; NRBC\%, percentage of nucleated red blood cells.

\section{Conformity rate of manual microscopic examination}

The paired Student's $t$-test (Table 3) was used to compare the WBC DIFF value and the NRBC count obtained with the BC-6800Plus and with manual microscopy. The Pearson correlation coefficient (r) between the BC-6800Plus results and the manual microscopy results ranged from 0.5771 (percentage of basophils) to 0.9146 (NRBC\%). The percentage of basophils calculated with the BC-6800Plus was significantly different from that obtained by manual counting $(\mathrm{P}<0.001)$. However, there were no significant differences between the two methods for the percentage of neutrophils (Neu\%), lymphocytes (Lym\%), monocytes (Mon\%), eosinophils (Eos\%), nor NRBC\% (P>0.05).

\section{Limit of quantitation}

The BC-6800Plus system showed a good limit of quantitation in the detection of nucleated red cells. It was able to detect 0.01 nucleated red cells out of 100 cells, with excellent performance parameters (Table 4).

\section{Discussion}

The rapid and accurate classification and reporting of hemocytes is a basic requirement of clinical hematology tests. Manual classification methods are gradually being replaced by automated blood cell analyzer machines, that can automatically classification blood cell types and are easier to standardize. In hospital hematology laboratories, analysis of blood cells are routinely used to accurately screen for pathological conditions $(1,17,18)$. These tests usually need to be verified through repeated microscopic examinations to ensure the accuracy of the report $(9,17,19)$. Therefore, blood cell analyzers need to be capable of quickly and accurately screening pathological specimens, 
A

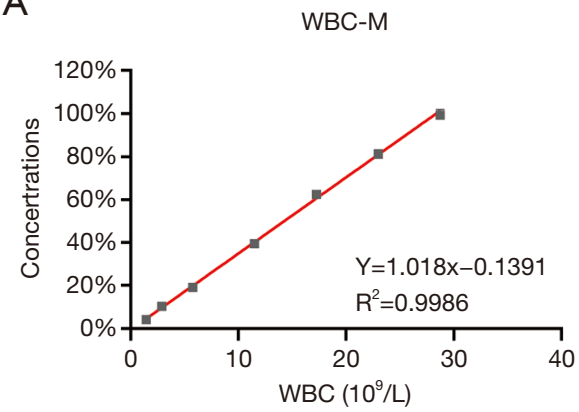

C

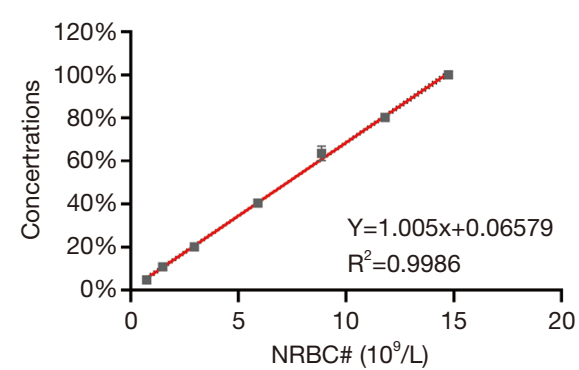

B

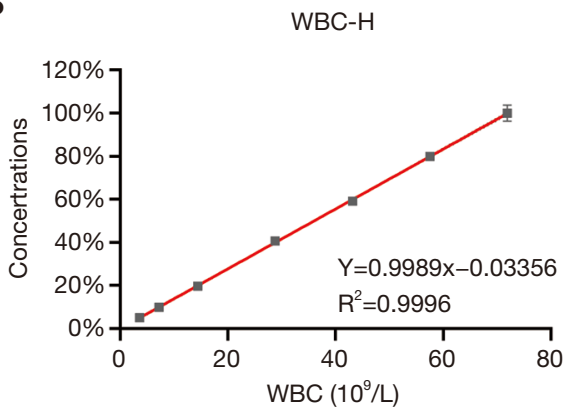

D

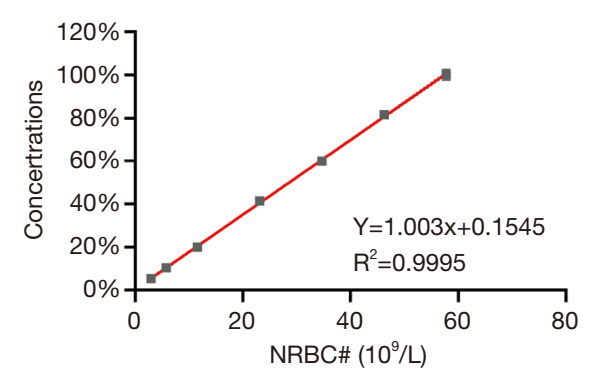

Figure 2 The figure shows that Mindray BC-6800Plus has a good linear relationship at medium and high concentrations. (A) The WBC showed good linear relationships at median concentrations; (B) the WBC showed good linear relationships at high concentrations; (C) the NRBC\# showed good linear relationships at median concentrations; (D) the NRBC\# showed good linear relationships at high concentrations. $M$, median value; $H$, high value; $\mathrm{WBC}$, white blood cell; NRBC\#, number of nucleated red blood cells.

reducing the workload of microscopic examination, and improving the detection rate under the pathological state. The BC-6800Plus is the latest blood cell analyzer from Mindray. This current study evaluated and validated the performance of the BC-6800Plus. The evaluation included the basic properties of the instrument, such as precision and carryover, and its clinical performance, including RBC count and NRBC count. The results indicated that the BC-6800Plus has outstanding performance, especially for the detection of NRBCs. It satisfied the requirements of accuracy of test results, rapid turnaround time of whole blood samples, and reduced the laboratory workload of microscopic examinations.

A low rate of carrying contamination is a basic performance characteristic of all blood cell analyzers. The results with the BC-6800 Plus demonstrated that high concentrations of WBCs and NRBCs did not increase the results of the low value specimens in subsequent tests.

The repeatability tests showed that BC-6800Plus had good performance in both low and high value samples. The percentage CV range for WBCs was 0.98-1.72, which was significantly lower than the manufacturer's advertised
$\mathrm{CV}$ of less than $4 \%$. Similarly, the percentage CV range for NRBC\# and NRBC\% was $0.62-6.97$ and 1.17-6.90, respectively, which were also significantly lower than the manufacturer's advertised CV of less than $20 \%$. In general, the BC-6800Plus showed good repeatability and accuracy in detecting WBCs and NRBCs. Due to the low detection value, the $\mathrm{CV}$ for the percentage of basophils was large, but the SD was very small, and thus, it still meets the requirements of conventional use.

By using high and median concentration samples to verify the linearity of BC-6800Plus, we obtained a WBC count range of $0.18-487.14 \times 10^{9}$ cells/L. There was a good linear relationship for NRBCs ( $\mathrm{r}$ value of NRBC\# and $\mathrm{NRBC} \%$ was 1.005 and 1.003 , respectively). The WBC count covers the vast majority of clinical specimens, while NRBC\# and NRBC\% can also be applied to the vast majority of clinical specimens. These results are beneficial for the diagnosis of blood system diseases and the detection of nucleated erythrocytes in peripheral blood. The superior linearity of the BC-6800Plus can reduce the manual effort required and avoid errors caused by manual dilutions.

The BC-6800Plus and the Sysmex XN-9100 systems 

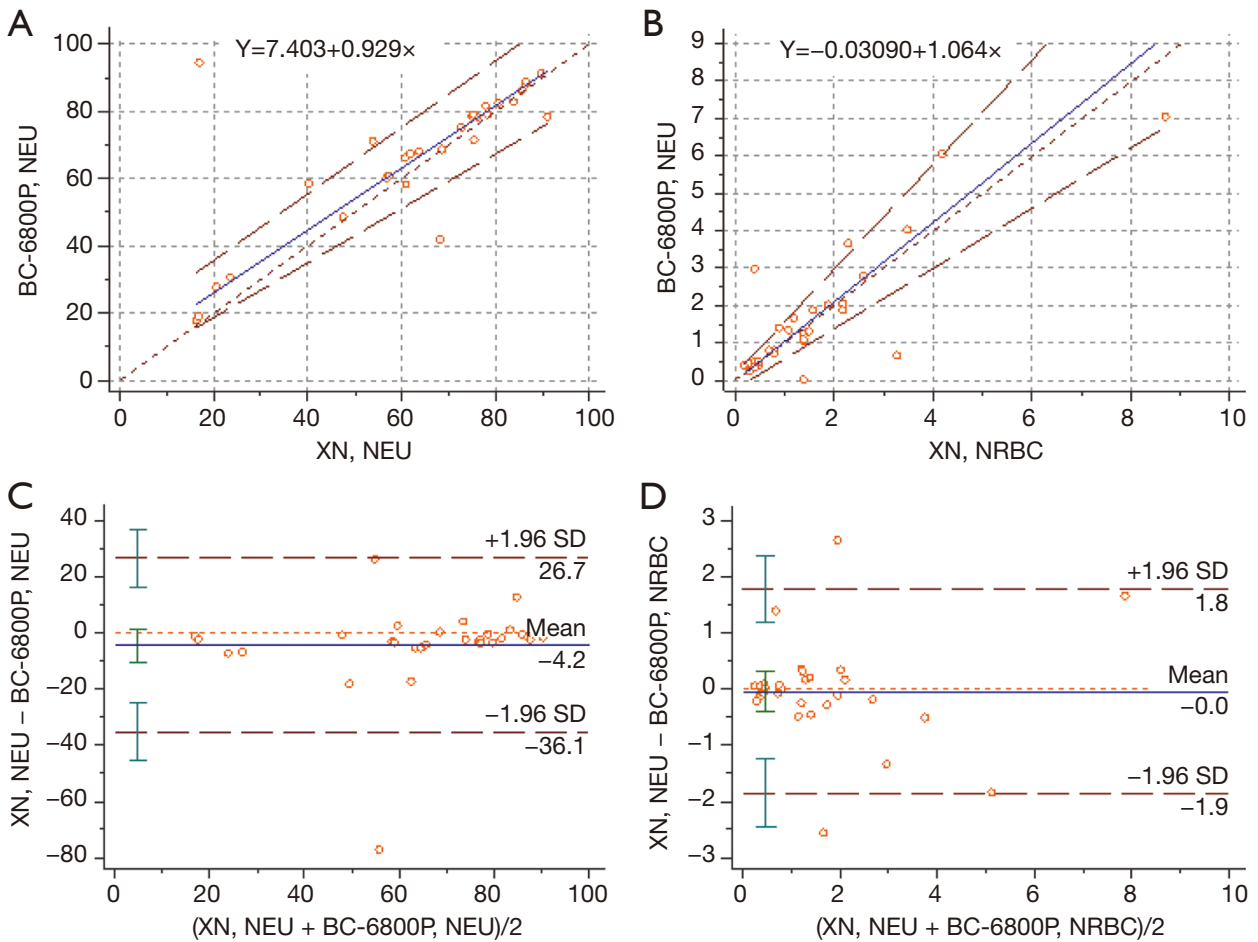

Figure 3 The figure shows that Mindray BC-6800Plus and Sysmex XN-9100 systems are highly consistent. (A) The Mindray BC-6800Plus and the Sysmex XN-9100 systems highly were correlated in NEU by Passing-Bablok regression, solid blue line represents Passing-Bablok fit, purple dotted line represents 95\% CI; (B) the Mindray BC-6800Plus and the Sysmex XN-9100 systems were highly correlated in NRBC by Passing-Bablok regression, solid blue line represents Passing-Bablok fit, purple dotted line represents 95\% CI; (C) the Mindray BC6800Plus and the Sysmex XN-9100 systems highly were correlated in NEU by Bland-Altman analyses; (D) the Mindray BC-6800Plus and the Sysmex XN-9100 systems highly were correlated in NRBC by Bland-Altman analyses. NEU, neutrophils; NRBC, number of nucleated red blood cells; SD, standard deviation.

Table 3 A comparison of WBC DIFFs and NRBC counts using the Mindray BC-6800Plus analyzer and manual counting methods

\begin{tabular}{|c|c|c|c|c|c|c|}
\hline Parameters & $\mathrm{N}$ & $\begin{array}{l}\text { Manual, mean } \\
\quad \text { (range) }\end{array}$ & $\begin{array}{l}\text { BC-6800Plus, mean } \\
\text { (range) }\end{array}$ & $\begin{array}{l}\text { Mean difference } \\
\quad(95 \% \mathrm{Cl})\end{array}$ & $\begin{array}{l}\text { Correlation } \\
\text { coefficient }\end{array}$ & $P$ value \\
\hline $\mathrm{Neu} \%$ & 723 & $69.242(0.00-99.96)$ & $69.400(6.80-97.30)$ & $0.156(-0.785$ to 1.096$)$ & 0.8023 & $<0.001$ \\
\hline Mon\% & 723 & $5.936(0.00-47.89)$ & $8.026(0.00-47.00)$ & 2.089 (1.747 to -2.432$)$ & 0.6952 & $<0.001$ \\
\hline Eos\% & 723 & $1.121(0.00-10.92)$ & $1.632(0.00-10.80)$ & $0.511(0.435$ to 0.587$)$ & 0.8365 & $<0.001$ \\
\hline NRBC\% & 690 & $2.557(0.00-53.98)$ & $3.266(0.01-59.18)$ & $-0.690(-0.889$ to -0.492$)$ & 0.9146 & $<0.001$ \\
\hline
\end{tabular}

WBC, white blood cell; DIFF, differential; NRBC, nucleated red blood cell; Cl, confidence interval; Neu\%, percentage of neutrophils; Lym\%, percentage of lymphocytes; Mon\%, percentage of monocytes; Eos\%, percentage of eosinophils; Bas\%, percentage of basophils; NRBC\%, percentage of nucleated red blood cells. 
Table 4 Limit of quantitation for the detection of NRBCs using the BC-6800Plus analyzer

\begin{tabular}{|c|c|c|c|c|c|c|}
\hline Parameters & \multicolumn{6}{|c|}{ Concentration } \\
\hline \multicolumn{7}{|c|}{ BC-6800Plus\#1 } \\
\hline 1 & 0.079 & 0.039 & 0.030 & 0.016 & 0.000 & 0.000 \\
\hline 2 & 0.045 & 0.054 & 0.027 & 0.012 & 0.000 & 0.000 \\
\hline \multicolumn{7}{|c|}{ BC-6800Plus\#2 } \\
\hline 1 & 0.056 & 0.031 & 0.016 & 0.010 & 0.000 & 0.000 \\
\hline 2 & 0.053 & 0.071 & 0.028 & 0.013 & 0.000 & 0.000 \\
\hline 3 & 0.049 & 0.053 & 0.016 & 0.012 & 0.000 & 0.000 \\
\hline
\end{tabular}

NRBC, nucleated red blood cell.

were comparable in terms of WBC counts and NRBC detection. Passing-Bablok and Bland-Altman analyses demonstrated that the two systems were highly correlated when analyzing the same sample. In terms of leukocyte classification, the BC-6800Plus and manual microscopy methods showed more consistent results. For the detection of NRBCs, the BC-6800Plus results showed a high correlation with manual microscopy results $(\mathrm{r}=0.9146)$. Therefore, BC-6800Plus showed good sensitivity and specificity for WBC classification and NRBC identification.

In conclusion, the Mindray BC-6800Plus blood cell analyzer demonstrated strong analytical capability and detection performance in clinical applications, especially in the detection of NRBCs. The BC-6800Plus provides a fast and accurate method for the analysis of blood samples and satisfies the clinical requirements of an efficient laboratory test.

\section{Acknowledgments}

Funding: None.

\section{Footnote}

Reporting Checklist: The authors have completed the MDAR reporting checklist. Available at https://dx.doi. org/10.21037/apm-21-1772

Data Sharing Statement: Available at https://dx.doi. org/10.21037/apm-21-1772

Conflicts of Interest: All authors have completed the ICMJE uniform disclosure form (available at https://dx.doi. org/10.21037/apm-21-1772). The authors have no conflicts of interest to declare.

Ethical Statement: The authors are accountable for all aspects of the work in ensuring that questions related to the accuracy or integrity of any part of the work are appropriately investigated and resolved. All procedures performed in this study involving human participants were in accordance with the Declaration of Helsinki (as revised in 2013). The study was approved by the Ethics Committee of the Guangyuan Central Hospital, Sichuan, China and informed consent was taken from all the patients.

Open Access Statement: This is an Open Access article distributed in accordance with the Creative Commons Attribution-NonCommercial-NoDerivs 4.0 International License (CC BY-NC-ND 4.0), which permits the noncommercial replication and distribution of the article with the strict proviso that no changes or edits are made and the original work is properly cited (including links to both the formal publication through the relevant DOI and the license). See: https://creativecommons.org/licenses/by-nc-nd/4.0/.

\section{References}

1. Morton SU, Brettin K, Feldman HA, et al. Association of nucleated red blood cell count with mortality among neonatal intensive care unit patients. Pediatr Neonatol 2020;61:592-7.

2. Wang Z, Cheng L, Sun Y, et al. Enhanced isolation of fetal 
nucleated red blood cells by enythrocyte-leukocyte hybrid membrane-coated magnetic nanoparticles for noninvasive pregnant diagnostics. Anal Chem 2021;93:1033-42.

3. Bergens MA, Pittman GS, Thompson IJB, et al. Smokingassociated AHRR demethylation in cord blood DNA: impact of CD235a+ nucleated red blood cells. Clin Epigenetics 2019;11:87.

4. Nombela I, Ortega-Villaizan MDM. Nucleated red blood cells: immune cell mediators of the antiviral response. PLoS Pathog 2018;14:e1006910.

5. Nitzan I, Kasirer Y, Mimouni FB, et al. Elevated nucleated red blood cells in neonates with down syndrome and pulmonary hypertension. J Pediatr 2019;213:232-4.

6. Narc H, Oktay MM, Ayrik C, et al. Nucleated red blood cells as predictor of all-cause mortality in emergency department. Am J Emerg Med 2021;46:335-8.

7. Houyhongthong V, Nunphuak W, Sripatumtong C, et al. Automated nucleated red blood cell count using the Mindray BC-6800 hematology analyzer. Int J Lab Hematol 2018;40:611-6.

8. Constantino BT, Rivera GKQ. Cutoff value for correcting white blood cell count for nucleated red blood cells: what is it? Why is it important? Lab Med 2019;50:e82-90.

9. Sheng N, Liu L, Liu H. Quantitative determination of agglutination based on the automatic hematology analyzer and the clinical significance of the erythrocyte-specific antibody. Clin Chim Acta 2020;510:21-5.

10. Kulik K, Kwiecień I, Chełstowska B, et al. Evaluation and comparison of the new Mindray BC-6200 hematology analyzer with ADVIA 2120i. Int J Lab Hematol 2021;43:395-402.

Cite this article as: Wang $\mathrm{N}$, Jiang J, Wang J, He Y, Wen N, Liu Y, Wang Y, Li H, Shi P. Detection of nucleated red blood cells using the Mindray BC-6800Plus hematology analyzer: a clinical performance evaluation. Ann Palliat Med 2021;10(8):88088817. doi: 10.21037/apm-21-1772
11. Gavriilaki E, Brodsky RA. Complementopathies and precision medicine. J Clin Invest 2020;130:2152-63.

12. Joyner MJ, Paneth N. Promises, promises, and precision medicine. J Clin Invest 2019;129:946-8.

13. Lila AM, Parfenov VA, Cattry E, et al. Pharmacokinetic linearity of naproxen and efficacy of naproxen sodium at various doses. Int J Clin Pharmacol Ther 2020;58:316-31.

14. Woo KS, Jeong IH, An GD, et al. Performance evaluation of new Abbott Alinity hq hematology analyzer. Int J Lab Hematol 2019;41:657-63.

15. Post A, Kaboré B, Reuling IJ, et al. The XN-30 hematology analyzer for rapid sensitive detection of malaria: a diagnostic accuracy study. BMC Med 2019;17:103.

16. Uemoto M, Makino M, Ota Y, et al. Determination of minor and trace metals in aluminum and aluminum alloys by ICP-AES; evaluation of the uncertainty and limit of quantitation from interlaboratory testing. Anal Sci 2018;34:719-24 .

17. Dufey F. Derivation of Passing-Bablok regression from Kendall's tau. Int J Biostat 2020. [Epub ahead of print]. doi: 10.1515/ijb-2019-0157.

18. Gerke O. Reporting standards for a Bland-Altman Agreement analysis: a review of methodological reviews. Diagnostics (Basel) 2020;10:334.

19. Slim CL, Wevers BA, Demmers MWHJ, et al. Multicenter performance evaluation of the Abbott Alinity hq hematology analyzer. Clin Chem Lab Med 2019;57:1988-98.

(English Language Editor: J. Teoh) 